\title{
Degree of Polarization for Weather Radars
}

\author{
Michele Galletti, Madhu Chandra, Thomas Börner \\ DLR - German Aerospace Agency \\ Microwaves and Radar Institute \\ Oberpfaffenhofen, Germany \\ e-mail: michele.galletti@dlr.de
}

\author{
David Henry Oliver Bebbington \\ University of Essex \\ Department of Electronic Systems Engineering \\ Colchester, Essex, UK
}

\begin{abstract}
Future operational weather radars are likely to implement hybrid polarization, an operating mode that involves transmitting $45^{\circ}$ slant polarization and receiving the horizontal and vertical components of the backscattered field. In this work, the degree of polarization at slant send is theoretically considered and experimentally evaluated from fully polarimetric signatures in order to assess its potential for use in next generation operational weather radars.
\end{abstract}

Keywords: Degree of Polarization, Instantaneous Scattering Matrix, Scattering Matrix, Depolarisation Response.

\section{INTRODUCTION}

Weather Radars are a fundamental tool for National Weather Services. Planned operational systems are likely to include Doppler and dual polarization, whose usefulness has been widely demonstrated in the last twenty years [1]. Such radars will probably implement hybrid polarization, a mode that involves transmitting slant $45^{\circ}$ and receiving the horizontal and vertical components of the backscattered signal. The reasons for such a choice are both theoretical and practical. The theoretical assumption is that weather targets most often appear to satisfy mirror reflection symmetry about the vertical axis. Practical considerations that make this choice operationally attractive is that, besides being more expensive, switched systems are characterized by increased feed complexity and more difficult calibration procedures. Further, hybrid polarization was conceived to effectively measure $Z_{\mathrm{DR}}$, KDP and $\rho_{\mathrm{HV}}$, which have been perceived operationally to be useful qualitative and quantitative parameters.

A variable available to dual polarization coherent radar systems is the degree of polarization, obtainable from Wolf's coherency matrix. The sensitivity of the degree of polarization to incoherent targets is however dependent on the transmit polarization state. To investigate this dependence, we resorted to process fully polarimetric weather radar signatures and compute the degree of polarization from the same dataset for different transmit states. A way to measure the degree of polarization capability to capture information from an incoherent target is a confrontation with entropy [4], a scalar quantity relating to the heterogeneity of statistically independent degrees of freedom existing in the scattering population. Entropy is a function of the eigenvalues of the covariance matrix and, contrary to the degree of polarization, can be obtained only from fully polarimetric signatures. The minimal degree of polarization (the degree of polarization obtained from the transmit states that minimize its value) should mirror the behavior of entropy rather faithfully.

A fully polarimetric radar is able to transmit pulses whose polarization state is switched every pulse repetition period (polarization agility) and is set to simultaneously receive the co- and cross- polar components of the backscattered signal (dual receiver). Such a set up allows quasi-simultaneous measurements of the complete scattering matrix. If a switched system is used and the complete scattering matrix is available, some more sophisticated signal processing approaches can be envisioned. Examples of these techniques include the rotation of the measured set of matrices to other polarization basis or the application of target decomposition theorems. The first meteorological radar designed to measure complete scattering matrices of weather targets was developed at DLR about twenty years ago and is known to the weather radar community as POLDIRAD, acronym for polarization diversity radar. The term polarization diversity refers to its capability of being able to fast-switch on transmit between any pair of orthogonal polarization states. For a detailed technical description of the system, we refer to [5]. To collect the data presented in this work, POLDIRAD was operated to switch between horizontal and vertical polarization states on transmit, and was set to receive the copolar and cross-polar components of the backscattered signal. This operation mode is called VHVH, but, as pointed out above, other modes are also possible like RLRL using right and left circularly polarized transmissions, as well as hybrid polarization. Ideally, all elements of a scattering matrix should be measured simultaneously. However, since (unless some coding scheme can be used) the transmit polarizations must be emitted sequentially, the scattering matrix measured by a fully polarimetric weather radar is affected by both mean motion of the target and decorrelation due to random displacements of the single scatterers. Special signal processing procedures were implemented to solve this problem [6].

\section{THEORY}

\section{A. Degree of Polarization}

Measurements done with a dual polarisation coherent receiver can be considered as samples of a random Jones vector of the form

$$
E(t)=\left[\begin{array}{l}
E_{1}(t) \\
E_{2}(t)
\end{array}\right]=\left[\begin{array}{l}
\left|E_{1}(t)\right| e^{i \varphi_{1}(t)} \\
\left|E_{2}(t)\right| e^{i \varphi_{2}(t)}
\end{array}\right]
$$




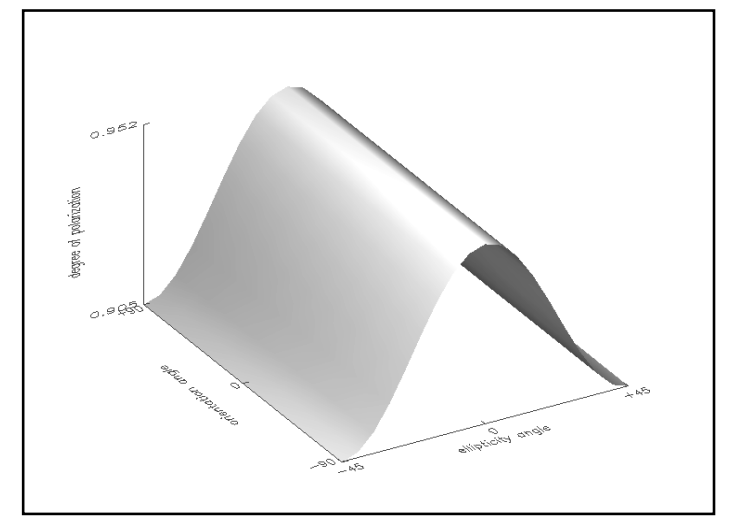

Figure 1. Depolarisation Response for a cloud of randomly oriented, slightly oblate, spheroids $\left(\mathrm{B}_{0}=0.05\right)$

The covariance of a random Jones vector (Wolf's coherency matrix $\mathrm{J}$ ) and the degree of polarization $\mathrm{p}$ read

$$
\begin{array}{r}
\operatorname{Cov}(E)=J=\left\langle E \cdot E^{+}\right\rangle \\
p=\sqrt{1-\frac{4 \operatorname{det}(J)}{(\operatorname{trace}(J))^{2}}}=\frac{\lambda_{1}-\lambda_{2}}{\lambda_{1}+\lambda_{2}}
\end{array}
$$

As the lambdas are Wolf's matrix eigenvalues, the degree of polarization $\mathrm{p}$ is a basis invariant quantity and as such does not depend on the orthogonal pair of polarimetric channels chosen to sample the backscattered wave. However, for weather radar applications, when we want to relate the degree of polarisation of the backscattered wave to some property of the illuminated target, a couple of theoretical remarks might be helpful. For a coherent target, the return is totally polarised, regardless of the transmit polarization state. For an incoherent target, the degree of polarisation of the backscattered wave does in general depend on the polarisation state of the transmitted wave. Such a function can be plotted directly on the Poincare sphere or with the help of surface plots, and could be referred to as the depolarization response of an incoherent target. We now consider two cases: Isotropic targets $\left(Z_{\mathrm{DR}}=0\right)$ and anisotropic targets $\left(\mathrm{Z}_{\mathrm{DR}}>0\right)$.

1) Isotropic targets $\left(Z_{D R}=0\right)$

A simple model for isotropic weather targets can be thought of as a cloud of randomly oriented spheroids. Considering Huynen parameters, a simple way to compute the degree of polarization as a function of the transmitted polarization state is obtained by considering the following Kennaugh matrix.

$$
\mathrm{K}_{\text {iso }}=\left[\begin{array}{cccc}
1+B_{0} & 0 & 0 & 0 \\
0 & 1 & 0 & 0 \\
0 & 0 & 1 & 0 \\
0 & 0 & 0 & -1+B_{0}
\end{array}\right]
$$

$\mathrm{B}_{0}$ ranges between 0 and 1 , depending if the spheroids are spheres $\left(\mathrm{B}_{0}=0\right)$ or dipoles $\left(\mathrm{B}_{0}=1\right)$. Simple algebra yields the following expression for the degree of polarization for a cloud of randomly oriented spheroids:

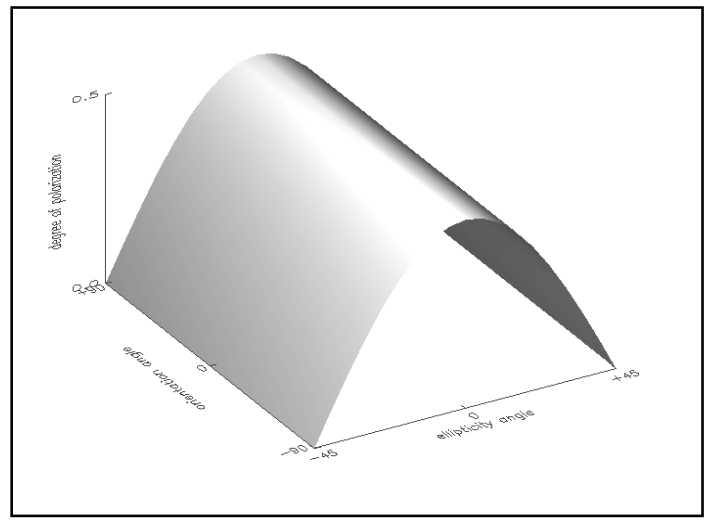

Figure 2. Depolarisation Response for a cloud of randomly oriented dipoles $\left(\mathrm{B}_{0}=1\right)$

$$
p=\frac{\sqrt{\cos ^{2}(2 \chi)+\left(1-B_{0}\right)^{2} \sin ^{2}(2 \chi)}}{1+B_{0}}
$$

Here, $\chi$ is the ellipticity angle and $\mathrm{B}_{0}$ is the generator of target structure. The above expression shows that, for an isotropic target, the degree of polarization attains its minimal values at the poles of the Poincare sphere and the maximal values at the equator (Fig. 1-2). Further, if we consider the quantity $1-p$, the relation between the minimum and the maximum is a simple $3 \mathrm{~dB}$ difference.

$$
1-p_{\min }=\frac{2 B_{0}}{1+B_{0}} ; \quad 1-p_{\max }=\frac{B_{0}}{1+B_{0}}
$$

The depolarization response of an isotropic target shows a number of symmetries, namely invariance with respect to orientation angle and handedness of the transmitted polarization state.

\section{2) Anisotropic Targets $\left(Z_{D R}>0\right)$}

In the case of rain a Kennaugh matrix representing a cloud of spherical plus a cloud of slightly oblate raindrops was constructed with the following values.

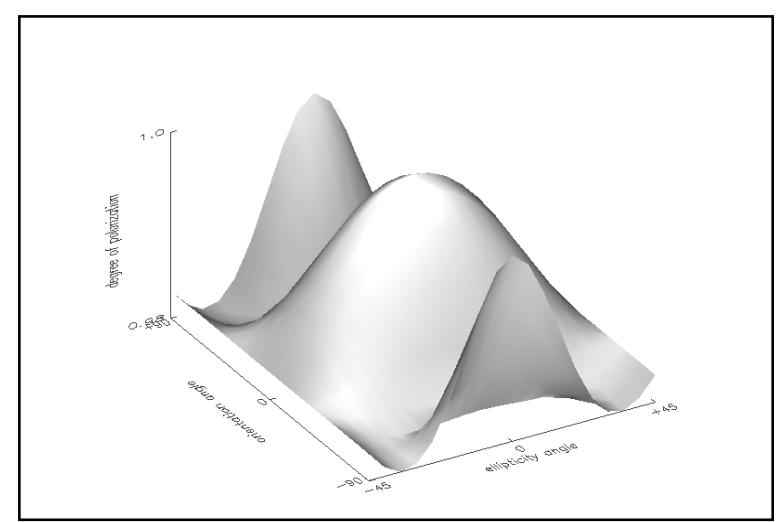

Figure 3. Depolarisation Response of a bimodal distribution of vertically aligned raindrops $\left(\mathrm{Z}_{\mathrm{DR}}=1.7 \mathrm{~dB}\right)$ 


$$
\mathrm{K}_{\text {rain }}=\left[\begin{array}{cccc}
1.0394 & 0.397 & 0 & 0 \\
0.397 & 1.0394 & 0 & 0 \\
0 & 0 & 0.9605 & 0 \\
0 & 0 & 0 & -0.9605
\end{array}\right]
$$

The depolarization response of this bimodal distribution has a different pattern than the isotropic case and is in general representative of anisotropic weather targets (rain). The degree of polarization attains its minimal values on the circular/slant circle of the Poincare sphere, and its maximal values at horizontal and vertical linear transmit (Fig. 3). In the case of rain, the degree of polarization at horizontal or vertical linear transmit (maximal degree of polarization) suffers from low dynamic range whereas at circular/slant send its capability of retrieving information is considerably enhanced.

If we assume that weather radar targets fall into one of the two above categories, the results can be summarized in the following points (DoP stands for Degree of Polarization):

- DoP at $\mathrm{H}$ or $\mathrm{V}$ send is always maximal

- DoP at circular (RHC or LHC) send is always minimal

- DoP at slant send is minimal for anisotropic and maximal for isotropic targets.

In the special case of radar meteorological applications, isotropic targets are generally characterised by more relevant depolarizing properties than anisotropic targets (graupel and hail depolarize more than rain). This is a fortunate circumstance as the degree of polarisation at slant send (that is minimal for anisotropic targets but maximal for isotropic targets) might have the potential to distinguish and retrieve information about a wide range of weather targets.

\section{B. Entropy-Alpha Decomposition}

In this section we refer to the Entropy/Alpha decomposition [3], [4]. For radar meteorological purposes, propagation is a relevant issue. Propagation through a medium can be described by a matrix $\mathrm{P}$ acting on a covariance matrix $\mathrm{C}$ (cross indicates adjoint) [2].

$$
C^{\prime}=P C P^{+}
$$

In the case of $\mathrm{P}$ being unitary, (8) reduces to a similarity transformation. Such a transformation set contains (in the strict sense) the set of polarization basis change, which, in turn, contains the set of rotations around the radar line of sight [3]. Since the eigenvalue problem is intrinsically invariant under unitary transformations, two observations can be made. The first is that, because of roll-invariance, whenever an anisotropic cloud of hydrometeors is illuminated, entropy is not dependent on the mean canting angle (insofar as only a rotation of the symmetry axis of the target occurs and the orientation distribution keeps unaltered). The second is that, since unitarity corresponds to energy conservation, propagation phenomena associated with non attenuating media with different electrical lengths at different polarizations do not affect entropy (in particular, entropy is not affected by differential phase shift). More specifically, the matrix P modeling propagation through a non-attenuating medium is an element of a representation of the rotation group $\mathrm{SO}(3)$, which is a subgroup of $\mathrm{U}(3)$. Nonattenuating propagation effects in the atmosphere map to $\mathrm{SL}(2, \mathrm{C})$ over $\mathrm{SU}(2)$ and the corresponding group representation is $\mathrm{SO}(3, \mathrm{C})$ which is isomorphic to the Lorentz group $\mathrm{SO}(3,1)$. Non-attenuating propagation effects map to a smaller set than $U(3)$ because the latter contains powerpreserving transformations that cannot physically occur in the atmosphere. Further, since entropy is explicitly normalized with respect to power, it is unaffected by polarization independent attenuation (but it is affected by differential attenuation). Entropy is an amplitude invariant scalar like $Z_{\mathrm{DR}}$ and LDR, but, unlike the latter, it has the property of being canting independent.

Alpha is an eigenvector-derived variable obtainable from Cloude's coherency matrix [3], [4]. It is roll-invariant but not differential propagation phase independent.

\section{DATA ANALYSIS}

From the same fully polarimetric dataset, we evaluated the degree of polarisation at horizontal, vertical, right hand circular, left hand circular, $+45^{\circ},-45^{\circ}$ slant polarisation and entropy. Between two antipodal transmit states the degree of polarisation does not show noticeable differences. Further, for the particular ray chosen, the difference between slant and circular is hardly noticeable and only the latter is displayed in the rayplot (left). The experimental results show that, for this particular ray (positive $\mathrm{Z}_{\mathrm{DR}}$ ), the degree of polarisation at slant (or circular) send carries the same information as entropy, and can effectively contribute to hydrometeor identification. Indeed, these variables show sensitivity to a number of different hydrometeor types like wet and dry snow, rain, graupel and hail (images not reported). Further, the data show these variables are experimentally robust against differential propagation phase effects. On the other hand, alpha appears to be severely affected by differential propagation phase effects that neatly dominate the retrieved signatures (compare $\Phi_{\mathrm{DP}}$ and $\alpha$ RHIs).

\section{CONCLUSIONS}

The degree of polarization at slant send is available to planned operational weather radars and its informationcarrying capability appears to be comparable with entropy. It should be noted that Entropy and the Degree of Polarisation carry a kind of information that is often similar but not necessarily identical to LDR that, by the way, is not available to one-shot systems. Entropy and the degree of polarization are experimentally robust against propagation effects and have good hydrometeor discrimination capabilities. These features prompt their use in Hydrometeor Classification Algorithms. Further, their potential to improve rain-rate retrieval algorithms need be further investigated [2]. Besides operational applications it should be emphasised their potential for cloud microphysics research (canting angles). On the other hand, $\alpha$ signatures appear to be dominated by differential propagation phase effects and similarities with $\Phi_{\mathrm{DP}}$ are relevant. Future work might be addressed at fully exploiting the potential of entropy and the degree of polarisation both for operational and cloud microphysics research applications. 


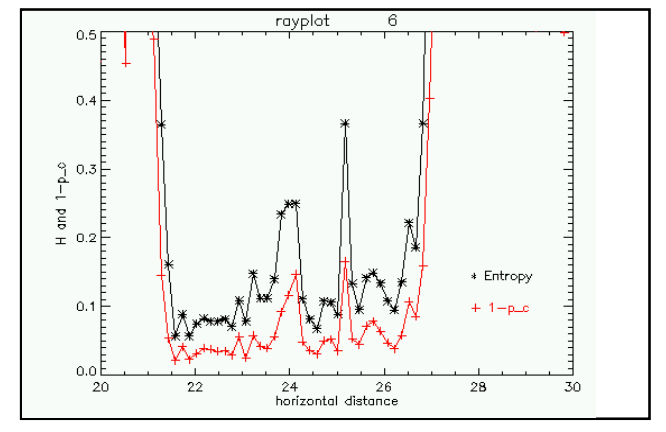

Figure 4. Minimal degree of polarization against entropy

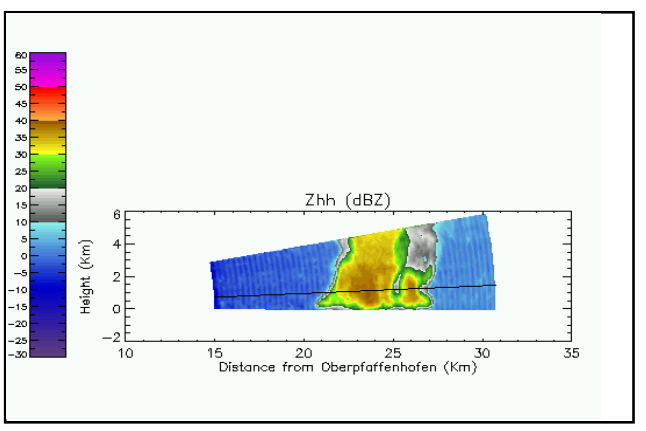

Figure 5. Reflectivity (dBZ)

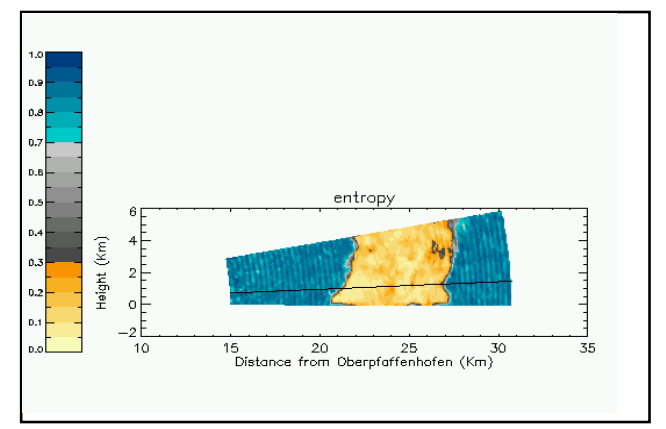

Figure 6. Entropy (Cloude Decomposition)

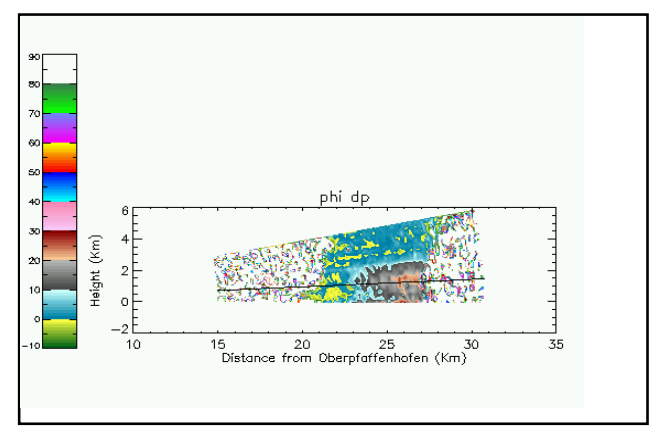

Figure 7. Differential Propagation Phase $\left(\Phi_{\mathrm{DP}}\right)$

\section{ACKNOWLEDGMENT}

This work was carried out under the aegis of the European Union funded project AMPER, Application of Multi-parameter Polarimetry in Environmental Remote Sensing.

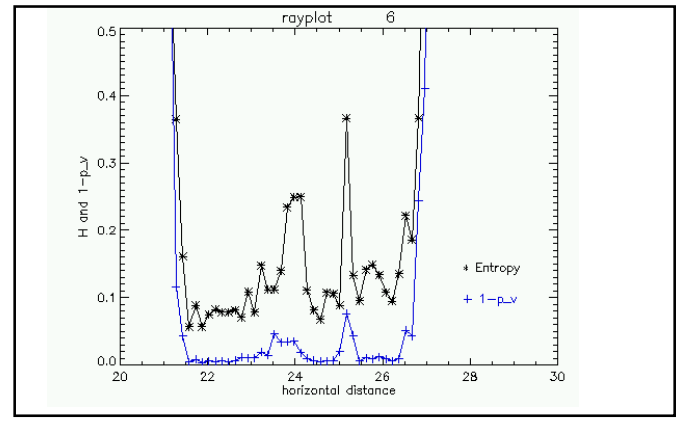

Figure 8. Maximal degree of polarization against entropy

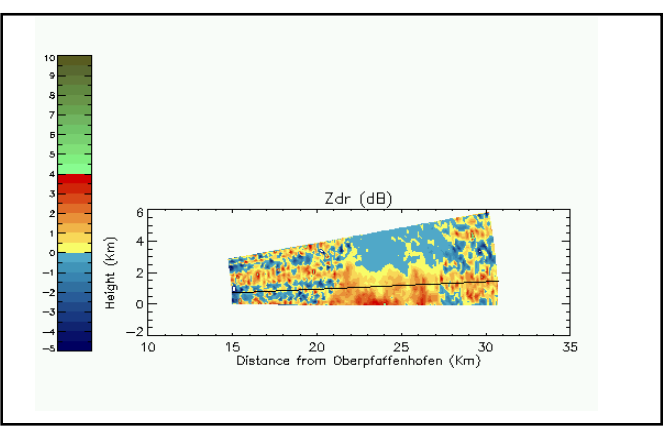

Figure 9. Differential Reflectivity (dB)

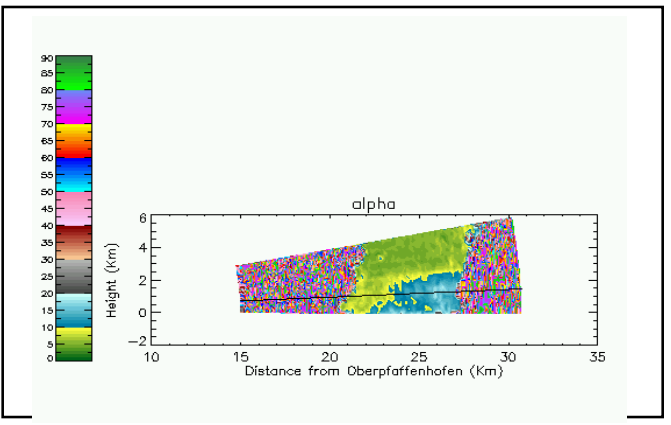

Figure 10. Alpha (Cloude Decomposition)

\section{REFERENCES}

[1] Bringi, V. N. and Chandrasekhar, V.: Polarimetric Doppler Weather Radar, Cambridge University Press, 2001.

[2] Bebbington, D.H.O., 'Degree of polarization as a radar parameter and its susceptibility to coherent propagation effects', URSI Commission F Symposium on Wave Propagation and Remote Sensing, Ravenscar, N. Yorkshire, UK, June 1992, 431-436.

[3] S. R. Cloude, E. Pottier,"A Review of Target Decomposition Theorems in Radar Polarimetry",IEEE Transactions on Geoscience and Remote Sensing, Vol. 34 No. 2, pp498-518, March 1996.

[4] S. R. Cloude, E. Pottier, "An Entropy Based Classification Scheme for Land Applications of Polarimetric SAR", IEEE Transactions on Geoscience and Remote Sensing, Vol. 35, No. 1, pp 68-78 ,January 1997.

[5] Schroth, A., M. Chandra, and Meischner, Peter F.: 'A C-band coherent polarimetric radar for propagation and cloud physics research', Journal of Oceanic and Atmospheric Technology, Vol. 5, pp 803-822, December 1988

[6] Chandrasekar, V., J. Hubbert, V. N. Bringi, and P. F. Meischner, 1992: Interpolation procedures to construct complete polarimetric signatures of distributed targets. Proc. SPIE, 1748, 200-212 